\title{
AVALIAÇÃO DO DESENVOLVIMENTO NEUROPSICOMOTOR DE CRIANÇAS NA PRIMEIRA INFÂNCIA EM UMA ESCOLA DE EDUCAÇÃO INFANTIL DO VALE DO RIO DOS SINOS - RS
}

\author{
NEUROPSYCHOMOTOR DEVELOPMENT ASSESSMENT OF CHILDREN IN FIRST CHILDHOOD IN A \\ SCHOOL OF EARLY CHILDHOOD EDUCATION AT VALE DO RIO DOS SINOS - RS
}

\author{
Simone de Paula ${ }^{a}$, Kétlin Caroline Griebeler ${ }^{b}$ \\ ${ }^{a}$ sdpaula@feevale.br, bketlincg@gmail.com \\ Universidade Feevale - Novo Hamburgo (RS), Brasil
}

Data de recebimento do artigo: 30/03/2017

Data de aceite do artigo: 07/08/2017

\section{RESUMO}

Introdução: $\mathrm{O}$ desenvolvimento infantil é um processo dinâmico que se inicia na vida intrauterina e envolve o crescimento físico, a maturação neurológica e a construção de habilidades comportamentais. Objetivos: Avaliar o desenvolvimento neuropsicomotor de crianças de até 24 meses, matriculadas em uma escola de educação infantil na região do Vale do Rio dos Sinos/RS. Materiais e métodos: Esta pesquisa consistiu em um estudo observacional descritivo com abordagem quantitativa. Participaram desta pesquisa 29 crianças, de ambos os gêneros, regularmente matriculadas em uma escola de educação infantil da região, com idades entre 1 e 24 meses (19,36 \pm 4,6 meses). Para a avaliação do desenvolvimento das crianças, foi utilizado o Teste de Triagem de Desenvolvimento de Denver II (TTDD II), que é composto por 125 itens distribuídos em quatro domínios: pessoal-social, motor fino-adaptativo, linguagem e motor grosso. Os itens foram interpretados como: normal, cautela e atraso. Resultados: De acordo com o TTDD II, $68,97 \%$ das crianças avaliadas apresentaram desenvolvimento infantil adequado e 31,03\% suspeito atraso no desenvolvimento. Quanto aos itens avaliados em cada área, o domínio da linguagem apresentou um percentual maior de itens em cautela ou atraso. Conclusóes: Por meio da interpretação dos resultados desta pesquisa, foi possível observar que as crianças, em sua maioria, apresentaram adequados padróes de desenvolvimento neuropsicomotor. Este estudo reforça a importância de estratégias de vigilância do desenvolvimento infantil que, certamente, refletirão na qualidade de vida destes indivíduos.

Palavras-chaves: Desenvolvimento infantil; educação infantil; saúde pública; intervenção precoce.

\section{ABSTRACT}

Introduction: The child development is a dynamic process that starts in the uterus and involves the physical growth, neurological maturation and behavioral skills building. Objectives: To evaluate the neurological development of children up to 24 months, enrolled in a school of early childhood education in Vale do Rio dos Sinos/RS. Materials and methods: This research consisted of a descriptive observational study with a quantitative approach. Twenty-nine children of both genders, regularly enrolled in a school of early childhood education in the region, aged between 1 and 24 months (19.36 \pm 4.6 months) participated in this study. For the developmental assessment of children, a screening test was used for the Denver II (DDST II), which is composed of 125 items distributed in 4 areas: personal-social, fine motor, language skills and gross motor. The items were interpreted as: normal, caution and delayed. Results: According to the DDST II, $68.97 \%$ of the evaluated children presented an appropriate child development and $31.03 \%$ of them presented a suspected delay in development. As to the items evaluated in each area, language domain presented great percentage of suspected delay or delay. Conclusions: Through the interpretation of the results of this research, it was possible to observe that most of the children presented adequate patterns of neuropsychomotor development. The present study reinforced the importance of child development surveillance strategies that will certainly reflect in these individuals' quality of life.

Keywords: Child development; child education; public health; early intervention. 


\section{Introdução}

O desenvolvimento infantil é um processo dinâmico que se inicia na vida intrauterina e envolve o crescimento físico, a maturaçáo neurológica e a construção de habilidades comportamentais que visam tornar a criança competente para responder às suas necessidades e as do seu meio ${ }^{1}$. Dentre as açóes do governo brasileiro, a vigilância ao desenvolvimento infantil constitui uma prioridade e compreende um eixo integrador da atenção à saúde da criança. Com o objetivo de promover e proteger a saúde da criança, desde a gestação até os 9 anos de vida, em agosto de 2015 o Ministério da Saúde aprovou a Política Nacional de Atenção Integral à Saúde da Criança (PNAISC) $^{2}$. A PNAISC destaca a prática de cuidado integral com ênfase na primeira infância através da interação de saberes multidisciplinares. O principal objetivo dessa política é promover o desenvolvimento integral da criança, contribuindo para um ambiente facilitador à vida com condiçóes dignas de existência e pleno desenvolvimento.

Os primeiros anos de vida de uma criança são fundamentais para o seu desenvolvimento. Considerado como "período crítico do desenvolvimento", essa etapa corresponde ao período de maior plasticidade neural, que é a capacidade do sistema nervoso central de reorganizar e adaptar as redes neuronais em resposta às exigências ambientais ou orgânicas ${ }^{3}$. Em virtude da explosão de sinapses e do rearranjo neuronal, diversos autores justificam que, nos primeiros anos de vida, as crianças podem desenvolver todas as suas potencialidades, sendo que abordagens pedagógicas, lúdicas ou terapêuticas são mais eficazes nesse período ${ }^{4}$.

Atualmente, devido às transformaçôes socioeconômicas da sociedade, é comum que a criança pequena fique a maior parte do dia em instituiçốes de educação infantil, o que torna as creches e pré-escolas cenários de destaque na promoção do desenvolvimento infantil 5 . Devido ao elevado tempo de permanência das crianças nesses locais, a identificação precoce de possíveis fatores de risco faz-se necessária para a prevenção de danos no neurodesenvolvimento a longo prazo, evitando problemas de coordenação, aprendizagem e socialização que se estendam até a fase adulta ${ }^{4,6}$.

Com base na importância de políticas públicas preventivas relacionadas à promoçáa do desenvolvimento normal e à detecção precoce de alteraçóes neuropsicomotoras no ambiente escolar, o objetivo geral deste estudo foi avaliar o desenvolvimento neuropsicomotor de crianças na primeira infância (até 24 meses) em uma escola de educaçáo infantil no Vale do Rio dos Sinos Rio Grande do Sul, Brasil.

\section{Metodologia}

Esta pesquisa caracterizou-se por um estudo observacional descritivo, com abordagem quantitativa. A coleta de dados foi realizada através do componente curricular Prática em Saúde Integral na Infância e Adolescência do curso de fisioterapia de uma universidade comunitária do Sul do Brasil, que atua na atenção primária a crianças e adolescentes de uma comunidade carente do Vale do Rio dos Sinos/RS.

Participaram desta pesquisa 29 crianças, de ambos os gêneros (19 meninos e 10 meninas), regularmente matriculadas em uma escola de educação infantil da região, com idades entre 1 a 24 meses $(19,36 \pm 4,6$ meses). Foram excluídas as crianças que tinham diagnóstico de desordens neurológicas ou que estavam pouco colaborativas no momento do teste.

Para a avaliação do desenvolvimento infantil, foi utilizado o Teste de Triagem de Desenvolvimento de Denver II (TTDD II), seguindo as instruçóes do manual do teste 7 . O teste é composto de 125 itens distribuídos em 4 domínios do desenvolvimento: pessoal-social, motor fino-adaptativo, linguagem e motor grosso. Após a identificação da faixa etária da criança, traçou-se uma linha vertical correspondente à idade. Foram avaliados todos os itens cortados pela linha, bem como os 3 itens totalmente à esquerda da linha em cada área de desenvolvimento. Os itens foram interpretados como: (N) normal, quando a criança realiza um item que foi cortado pela linha; (C) cautela, quando a criança falha em um item no qual a linha da idade cruza entre 75 a $90 \%$; e (A) atraso, quando a criança falha em um item que está totalmente à esquerda da linha da idade. Após interpretar cada item, o escore final do teste determinou duas classificaçóes: desenvolvimento adequado, quando não há atrasos ou no máximo 1 cautela; e desenvolvimento suspeito, quando há 2 ou mais cautelas e/ou 1 ou mais atrasos.

As avaliaçóes do desenvolvimento foram realizadas no ano de 2016 por acadêmicos do curso de fisioterapia, que foram submetidos a treinamento prévio para a utilização do teste. As crianças foram avaliadas individualmente em uma sala na escola, e os materiais utilizados foram: blocos de madeira quadrados e coloridos, uvas passas, lápis, boneca de plástico, canecas e papel branco. As professoras responsáveis participaram da aplicação e auxiliaram no relato de algumas atividades previstas no teste.

A permissão para iniciar o trabalho na creche foi concedida pela diretora e o acesso às crianças se deu após a assinatura do Termo de Consentimento Livre e Esclarecido (TCLE) pelos seus pais ou responsáveis. A pesquisa foi aprovada pelo Comitê de Ética da instituição 
(CAEE 4.08.03.07.779) e seguiu a Resolução 466 de 2012 do Conselho Nacional de Saúde.

Os dados foram analisados quantitativamente por meio de estatística descritiva e apresentados em números absolutos e percentuais, sob a forma de gráficos e tabelas, considerando-se os critérios de interpretação dos resultados do teste. $\mathrm{O}$ teste exato de Fisher também foi utilizado para analisar as diferenças entre os gêneros, tendo como base um nível de significância de 5\%.

\section{Resultados}

Após a realização do teste em ambiente escolar, observou-se que a maioria das crianças avaliadas $(n=20$; $68,97 \%$ ) apresentou um desenvolvimento infantil adequado, de acordo com a classificação global do TTDD II. Um percentual de 31,03\% ( $\mathrm{n}=9$ ) das crianças avaliadas recebeu a classificação de desenvolvimento suspeito, com 2 ou mais cautelas e/ou, pelo menos, um atraso (Gráfico 1). Somente 4 crianças (13,79\%) apresentaram mais de 1 atraso nos itens avaliados nos domínios do teste.

Gráfico 1: Classificação global do desenvolvimento infantil, de acordo com o Teste de Triagem de Denver II.

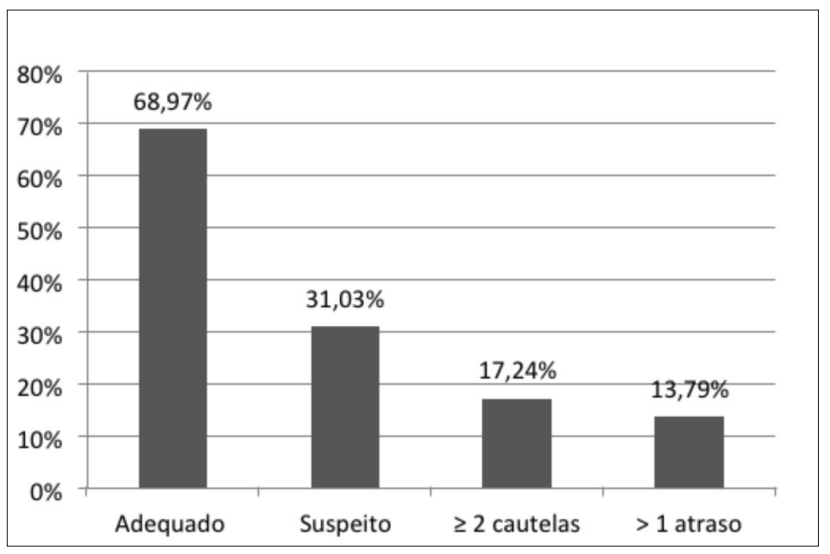

Fonte: Elaborado pelos autores, 2017.

Quando realizada a comparação por gênero, o escore final do TTDD II mostrou que 73,68\% dos meninos e $60 \%$ das meninas apresentaram um desenvolvimento neuropsicomotor adequado para a idade cronológica. Houve um percentual maior de meninas (40\%) classificadas com suspeita para atraso no desenvolvimento (Gráfico 2). No entanto, o teste exato de Fisher mostrou que não houve diferença estatisticamente significativa entre os grupos analisados $(\mathrm{p}=0,67)$.
Gráfico 2: Classificação global do desenvolvimento infantil por gênero, de acordo com o Teste de Triagem de Denver II.

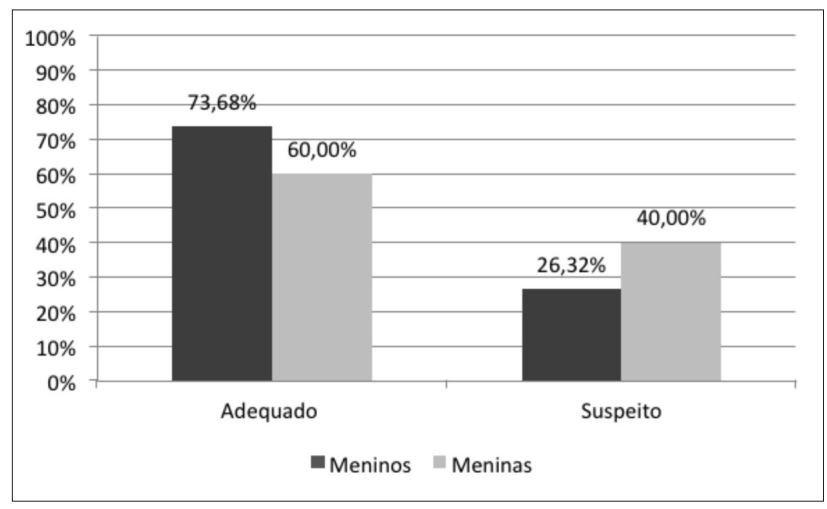

Fonte: Elaborado pelos autores, 2017.

$\mathrm{Na}$ análise das atividades específicas, foram identificados 12 itens em atraso (Tabela 1), distribuídos nos quatro domínios do TTDD II. O domínio da linguagem apresentou um percentual maior de itens em atraso $(\mathrm{n}=9 ; 75 \%)$, seguido pelos domínios motor grosso $(n=2 ; 16,66 \%)$ e pessoal-social $(n=1$; $8,3 \%)$. Já na avaliação do domínio motor grosso e pessoal-social, os itens "fica em pé sozinho" e "bebe de um copo", respectivamente, foram as principais atividades em atraso.

Tabela 1: Número de itens classificados em atraso, de acordo com os quatro domínios do Teste de Triagem de Denver II.

\begin{tabular}{lcc} 
Áreas & $\begin{array}{c}\text { Número de itens classificados em } \\
\text { atraso }\end{array}$ \\
& n & $\%$ \\
Pessoal-social & 1 & 8,3 \\
Motor fino-adaptativo & 0 & 0 \\
\hline Linguagem & 9 & 75 \\
Motor grosso & 2 & 16,66 \\
Total & 12 & 100 \\
\hline
\end{tabular}

Fonte: Elaborado pelos autores, 2017.

Em relação às crianças que apresentaram itens em cautela, foram identificadas 22 atividades que receberam essa classificação no momento do teste. O domínio linguagem também se destacou com um percentual de $54,54 \%$ de itens considerados como cautela, ou seja, quando a criança não executa uma atividade que é feita por 75 a $90 \%$ das crianças naquela faixa etária (Tabela 2). As principais habilidades que receberam a classificação cautela estiveram relacionadas, especialmente, à capacidade de falar uma ou mais palavras. 
Tabela 2: Número de itens classificados como cautela, de acordo com os quatro domínios do Teste de Triagem de Denver II.

\begin{tabular}{lcc} 
& Número de itens classificados como \\
Áreas & \multicolumn{2}{c}{ cautela } \\
& n & $\%$ \\
\hline Pessoal-social & 6 & 27,27 \\
Motor fino-adaptativo & 3 & 13,63 \\
\hline Linguagem & 12 & 54,54 \\
Motor grosso & 1 & 4,5 \\
Total & 22 & 100 \\
\hline
\end{tabular}

Fonte: Elaborado pelos autores, 2017.

\section{Discussão}

Este estudo apresentou uma prevalência de 68,97\% de crianças com adequado desenvolvimento infantil e $31,03 \%$ de crianças com suspeita para atrasos. Esses resultados corroboram com estudos realizados em crianças brasileiras $^{8-12}$. Em uma pesquisa semelhante desenvolvida na cidade de Canoas/RS ${ }^{11}$, os autores observaram uma prevalência de $27 \%$ de suspeita de atraso no desenvolvimento neuropsicomotor. De acordo com a análise multivariada do estudo, os fatores associados à suspeita de atraso no desenvolvimento foram: baixa renda familiar, gestação materna com intervalo interpartal inferior a 18 meses e mães sem o apoio dos pais da criança. De forma similar, um estudo de coorte também realizado no Sul do país ${ }^{13}$ demonstrou que a renda familiar inferior a um salário mínimo constituiu um fator de risco para alteraçôes do desenvolvimento infantil.

O crescimento e o desenvolvimento infantis são produtos multifatoriais associados aos aspectos ambientais, socioeconômicos e biológicos ${ }^{14}$. Nesse contexto, Martins et al. ${ }^{13}$ reforçam a importância de examinar o efeito da presença simultânea de múltiplos fatores de risco, tanto biológicos como ambientais. A renda familiar é determinante para a qualidade de vida das famílias, uma vez que as dificuldades constantes associadas à pobreza prejudicam o bem-estar psicológico dos pais e desfavorecem o ambiente interpessoal em casa.

Em relação ao gênero, este estudo não encontrou diferenças significativas entre meninos e meninas. De forma similar, Ozkan et al. ${ }^{15}$ e Rezende et al. ${ }^{10,16}$ também demonstraram que não houve diferenças em relaçáo ao gênero, considerando os resultados do Teste de Triagem de Denver II em pré-escolares. No entanto, alguns estudos têm postulado que as alteraçóes no neurodesenvolvimento são mais comuns em meninos. $\mathrm{O}$ resultado das análises da pesquisa de Brito et al. ${ }^{17}$ demonstrou que ser do sexo masculino se associou significativamente a um desempenho anormal no desenvolvimento. A influência do gênero nas aquisiçôes neuropsicomotoras na infância ainda não está clara e bem estabelecida.

$\mathrm{Na}$ análise das atividades nos quatro domínios do TTDD II, a linguagem apresentou um percentual maior de itens classificados em atraso. Esses resultados são condizentes com alguns estudos realizados em creches brasileiras $^{16-20}$ que demonstram um menor desempenho de crianças na primeira infância no domínio da comunicação. Nesse domínio, são avaliadas habilidades que variam desde a vocalização e a capacidade de imitar sons até a nomeaçãoo de figuras familiares e a emissão de frases curtas, dependendo da idade cronológica da criança. Nesta pesquisa, pode-se observar que a maioria das habilidades em atraso do domínio linguagem se encontravam na faixa etária dos 18 meses e estavam relacionadas, especialmente, à capacidade de falar uma ou mais palavras. A aquisição e o desenvolvimento da linguagem são processos evolutivos cujo período crítico acontece na faixa etária de zero a seis anos Entre 22 e 36 meses de idade, as crianças apresentam uma "explosão" na aquisição do vocabulário e passam a organizar essas palavras em frases para conseguirem comunicar seus desejos e suas necessidades. Elas aprendem palavras que são nomes de objetos ou pessoas e aquelas ligadas a relacionamentos sociais. ${ }^{21}$ Alguns autores referem que a criança passa por duas fases do desenvolvimento: pré-linguística e linguística. A pré-linguística é a fase das vocalizaçóes e acontece até os 11-12 meses de vida. A linguística refere-se à fase em que a criança começa a falar as primeiras palavras com compreensão e a comunicação intencional ganha um impulso significativo. Com o passar do tempo, a criança progride em relação à complexidade da expressão, de forma contínua, ordenada e sequencial. ${ }^{22}$

Investigaçóes recentes têm mostrado que o desenvolvimento linguístico pode apresentar alteraçóes significativas em razão de fatores de risco biológicos e socioambientais, resultando em alterações nos padrōes de aquisição da linguagem ${ }^{14,23}$. A baixa condiçáo socioeconômica, a idade e a escolaridade materna são alguns dos fatores de risco para atrasos fonológicos devido à diminuição do repertório linguístico, à baixa estimulação da fala e à priorizaçáo do cuidado voltado às necessidades básicas da criança ${ }^{19,24,25}$. Além disso, estudos também demonstram a importância de crianças em fase pré-escolar frequentarem instituiçóes de educação infantil para a aquisição de habilidades de comunicação. Nesses ambientes, os estímulos e as atividades lúdicas podem contribuir de forma significativa para o desenvolvimento da linguagem ${ }^{26}$. Lima et al..$^{25}$ acrescentam que as condiçóes das instituiçóes de educação infantil, a proporção educadora/criança, a qualificação das educadoras, a qualidade e estrutura do local e o tempo de permanência da criança são fatores determinantes para 
o desempenho de padróes de atividades e estímulos, assim como a estabilidade emocional da criança.

Além de déficits de aquisição na linguagem, atrasos nos domínios pessoal-social e motor grosso também são referenciados em estudos com crianças na primeira infância, em proporçõos menores, similarmente aos resultados encontrados neste estudo ${ }^{10,17,27}$. A independência, a interação com as outras pessoas e as habilidades motoras (como equilíbrio e coordenaçáo) são avaliadas nesses domínios através de itens como "ajuda em casa", "remove roupas", "bebe de um copo" e "fica em pé sozinho". $\mathrm{Na}$ área pessoal-social, valoriza-se a conquista da independência da criança para realizar tarefas da vida diária como lavar as máos, alimentar-se e escovar os dentes, por exemplo. Em virtude disso, as creches e pré-escolas devem criar condiçôes para que as crianças se interessem progressivamente pelo cuidado com o próprio corpo, executando açôes relacionadas à saúde e à higiene. No tocante ao comportamento motor, deve-se considerar que a interação dos aspectos físicos e estruturais do indivíduo com o ambiente em que está inserido e com a tarefa a ser aprendida são determinantes na aquisição e no refinamento das diferentes habilidades motoras. Aspectos da socialização e do controle motor postural são requisitos fundamentais para a exploração do ambiente e a aquisiçáo de habilidades cognitivas a longo prazo. ${ }^{16}$ Martín et al. $(2014)^{28}$ acrescentam que atrasos no desenvolvimento da linguagem e em outros domínios têm influência significativa na socialização, no comportamento, no aprendizado e, até mesmo, na empregabilidade. Conceitualmente, ressalta-se que o desenvolvimento neuropsicomotor é um processo amplo e não-fragmentado, dinâmico e contínuo, que expressa a conquista das habilidades mentais e físicas, a organização da personalidade e tudo o que permite ao indivíduo incorporar-se de forma ativa e transformadora à sociedade ${ }^{29}$.

Nesse contexto, a vigilância do desenvolvimento compreende todas as atividades relacionadas à promoção do desenvolvimento normal e à detecção de problemas durante a atenção primária à saúde da criança. Além disso, esse processo envolve profissionais da saúde, pais e professores, além de inserir todos esses elementos em um contexto sociocultural ${ }^{30}$. O Teste de Triagem de Denver II é o principal instrumento de avaliação do desenvolvimento infantil utilizado no mundo todo. No Brasil, além de ser uma ferramenta frequentemente utilizada em pesquisas para avaliaçáo do desenvolvimento de pré-escolares, foi uma das referências que nortearam a elaboração da Ficha de Avaliação do Desenvolvimento, do Ministério da Saúde ${ }^{31}$. Apesar da equivalência do teste em relação às crianças brasileiras e americanas, pesquisas que utilizam o Teste de Triagem de Denver II para o acompanhamento do desenvolvimento demonstram que esse instrumento pode apresentar resultados heterogêneos, a depender do contexto e da regiáo em que as crianças avaliadas estáo inseridas ${ }^{17}$. Por fim, ressalta-se que este é um teste de triagem e não visa a obtenção de diagnósticos clínicos. Logo, as crianças que apresentaram desempenho anormal neste estudo realizaráo avaliaçóes complementares para uma conclusão diagnóstica e multidisciplinar.

Embora haja limitaçóes neste estudo, tais como o pequeno número amostral e a ausência de associaçóes entre fatores de risco e atraso no desenvolvimento, é importante enfatizar que os resultados das análises realizadas nesta investigação proporcionam reflexão sobre os estímulos adequados em ambiente escolar e fornecem subsídios para a importância da atenção primária à saúde da criança em diferentes contextos.

\section{Conclusão}

Por meio da análise e interpretação dos resultados dessa pesquisa, foi possível observar que as crianças avaliadas apresentaram, em sua maioria, adequados padróes de desenvolvimento neuropsicomotor. Apesar disso, aspectos como a linguagem, a socialização e o desenvolvimento de algumas habilidades motoras apresentaram um percentual de suspeita para atraso no desenvolvimento. Em virtude de diversos fatores envolvidos na aquisiçáo de habilidades motoras e cognitivas na primeira infância, enfatiza-se com este trabalho a importância da abordagem intersetorial e interdisciplinar entre o sistema de saúde e a educação, especialmente em populaçóes mais vulneráveis. Portanto, as açôes de saúde devem transcender o acompanhamento do crescimento de crianças, evoluindo para estratégias de vigilância do desenvolvimento infantil que, certamente, refletirão na qualidade de vida e em melhores oportunidades socioeducacionais a longo prazo.

\section{Referências}

1. Miranda LP, Resegue R, Figueiras ACM. A criança e o adolescente com problemas do desenvolvimento no ambulatório de pediatria. Jornal de Pediatria. 2003;79(Supl 1):S33-S42.

2. BRASIL. Ministério da Saúde. Portaria no 1130 , de 5 de agosto de 2015. Institui a Política Nacional de Atenção Integral à Saúde da Criança (PNAISC) no âmbito do Sistema Único de Saúde (SUS). Diário Oficial da União, DF, 6 ago. 2015. Disponível em: <https://goo.gl/87X6aF>. Acesso em: 13 dez. 2017.

3. Johnston MV. Plasticity in the developing brain: implications for rehabilitation. Dev Disabil Res Rev. 2009;15(2):94-101.

4. Willrich A, Azevedo CCF, Fernandes JO. Desenvolvimento motor na infância: influência dos fatores de risco e programas de intervenção. Rev Neurocienc. 2009;17(1):51-6. 
5. Alves RCP, Veríssimo MLOR. Os educadores de creche e o conflito entre cuidar e educar. Rev Bras Crescimento Desenvolv Hum. 2007;17(1):13-25.

6. Baltieri L, Santos DCC, Gibim NC, Souza CT, Batistela ACT, Tolocka RE. Motor performance of infants attending the nurseries of public day care centers. Rev Paul Pediatr. 2010;28(3)283-9.

7. Frankenburg WK, Dodds J, Archer P, Shapiro H, Bresnick B, Maschka P, et al. Denver II: training manual. Denver: Denver Developmental Materials; 1992.

8. Carneiro JM, Brito APB, Santos MEA. Avaliação do desenvolvimento de crianças de uma creche através da escala de Denver II. REME Rev Min Enferm. 2011;15(2):174-80.

9. Moraes MW, Weber APR, Santos MCO, Almeida FA. Teste de Denver II: avaliação do desenvolvimento de crianças atendidas no ambulatório do Projeto Einstein na Comunidade de Paraisópolis. Einstein. 2010;8(2):149-53.

10. Rezende MA, Costa PS, Pontes PB. Triagem de desenvolvimento neuropsicomotor em instituiçôes de educação infantil segundo o Teste de Denver II. Esc Anna Nery Rev Enferm. 2005;9(3):348-55.

11. Pilz EML, Schermann LB. Determinantes biológicos e ambientais no desenvolvimento neuropsicomotor em uma amostra de crianças de Canoas/RS. Ciênc Saúde Coletiva. 2007;12(1):181-90.

12. Veleda AA, Soares MCF, Cézar-Vaz MR. Fatores associados ao atraso no desenvolvimento em crianças, Rio Grande, Rio Grande do Sul, Brasil. Rev Gaúch Enferm. 2011;32(1):79-85.

13. Martins MFD, Costa JSD, Saforcada ET, Cunha MDC. Qualidade do ambiente e fatores associados: um estudo em crianças de Pelotas, Rio Grande do Sul, Brasil. Cad Saúde Pública. 2004;20(3):710-8.

14. Neves KR, Morais RLS, Teixeira RA, Pinto PAF. Growth and development and their environmental and biological determinants. J Pediatr (Rio J). 2016;92(3):241-50.

15. Ozkan M, Senel S, Arslan EA, Karacan CD. The socioeconomic and biological risk factors for developmental delay in early childhood. Eur J Pediatr. 2012;171(12):1815-21.

16. Rezende MA, Beteli VC, Santos JLF. Avaliação de habilidades de linguagem e pessoal-sociais pelo Teste de Denver II em instituiçôes de educação infantil. Acta Paul Enferm. 2005;18(1):56-63.

17. Brito CML, Vieira GO, Costa MCO, Oliveira NF. Desenvolvimento neuropsicomotor: o teste de Denver na triagem dos atrasos cognitivos e neuromotores de pré-escolares. Cad Saúde Pública. 2011;27(7):1403-14.

18. Pinto FCA, Isotani SM, Sabatés AL, Perissinoto J. Denver II: comportamentos propostos comparados aos de crianças paulistanas. Rev CEFAC. 2015;17(4):1262-9.
19. Costa EF, Cavalcante LIC, dell'Aglio DD. Perfil do desenvolvimento da linguagem de crianças no município de Belém, segundo o Teste de Triagem de Denver II. Rev CEFAC. 2015;17(4):1090-102.

20. Ribeiro DG, Perosa GB, Padovani FHP. Fatores de risco para o desenvolvimento de crianças atendidas em Unidades de Saúde da Família, ao final do primeiro ano de vida: aspectos sociodemográficos e de saúde mental materna. Ciênc saúde coletiva. 2014;19(1):215-26.

21. Pedromônico MRMA, Affonso LA, Sañudo, A. Vocabulário expressivo de crianças entre 22 e 36 meses: estudo exploratório. Rev bras crescimento desenvolv hum. 2002;12(2):13-22.

22. Silva LK, Labanca L, Melo EMC, Costa-Guarisco LP. Identificação dos distúrbios da linguagem na escola. Rev CEFAC. 2014;16(6):1972-79.

23. Carniel CZ, Furtado MCC, Vicente JB, Abreu RZ, Tarozzo RM, Cardia SETR, et al. Influência de fatores de risco sobre o desenvolvimento da linguagem e contribuições da estimulação precoce: revisão integrativa da literatura. Rev CEFAC. 2017;19(1):109-18.

24. Muluk NB, Bayoğlu B, Anlar B. Language development and affecting factors in 3- to 6-year-old children. Eur Arch Otorhinolaryngology. 2014;271(5):871-8.

25. Lima SS, Cavalcante LIC, Costa EF. Triagem do desenvolvimento neuropsicomotor de crianças brasileiras: uma revisão sistemática da literatura. Fisioter Pesqui. 2016;23(3):336-42.

26. Mendes JCP, Pandolfi MM, Carabetta V Jr, Novo NF, Colombo-Souza P. Fatores associados a alteraçáo da linguagem em crianças pré-escolares. Rev soc bras fonoaudiol. 2012;17(2):177-81.

27. Rezende MA, Beteli VC, Santos JLF. Acompanhamento das habilidades motoras de crianças que frequentam creches e pré-escolas. Rev Latinoam Enferm. 2005;13(5):619-25.

28. Martín Ruiz ML, Valero Duboy MÁ, Torcal Loriente C, Pau de la Cruz I. Evaluating a web-based clinical decision support system for language disorders screening in a nursery school. J Med Internet Res. 2014;16(5):e139.

29. Gannam SS. Percepção de pais e professores do desenvolvimento de crianças de três a seis anos comparada com o Teste de Denver II [dissertação]. São Paulo: Universidade de São Paulo; 2009.

30. Organização Pan-Americana da Saúde. Manual para vigilância do desenvolvimento infantil no contexto da AIDPI. Washington, DC: OPAS; 2005.

31. Brasil. Ministério da Saúde. Secretaria de Políticas de Saúde. Área Técnica de Saúde da Criança. Fundamentos técnico-científicos e orientaçóes práticas para o acompanhamento do crescimento e desenvolvimento. Brasília, DF: Ministério da Saúde; 2000.

\section{Como citar este artigo:}

Paula S, Griebeler KC. Avaliação do desenvolvimento neuropsicomotor de crianças na primeira infância em uma escola de educação infantil do Vale do Rio dos Sinos - RS. Rev. Aten. Saúde. 2017;15(54):49-54. 